\title{
CONSUMO DE MEDICAMENTOS EN PROFESIONALES Y TÉCNICOS/ ADMINISTRATIVOS DE LA SALUD: SITUACIÓN DE PRESCRIPCIÓN
}

\author{
CONSUMPTION OF MEDICINES IN HEALTH PROFESSIONALS AND TECHNICAL/ \\ ADMINISTRATIVE STAFF: PRESCRIPTION SITUATION
}

CONSUMO DE DROGA NA PROFISSIONAL DE SAÚDE, TÉCNICOS/

ADMINISTRATIVOS: SITUAÇÃO DE PRESCRIÇÃO

\section{Edith Rivas Riveros}

Universidad de La Frontera. Temuco, Chile.

edith.rivas@ufrontera.cl

ORCID: 0000-0002-9852-4534

\section{Angélica Rivas Lienqueo}

Universidad Autónoma de Chile. Teтисо, Chile.

ORCID: 0000-0002-2939-351X

\section{Luis Bustos Medina}

CIGES. Departamento de Salud Pública. Universidad de La Frontera. Temuco, Chile. ORCID: 0000-0002-6917-3239

DOI: http://doi.org/10.22235/ech.v7i2.1652

Recibido: 13/07/2017

Aceptado: 22/04/2018

Resumen: El objetivo del estudio fue establecer la situación de consumo de medicamentos no prescritos en profesionales, técnicos/administrativos de salud y los factores de riesgo asociados. Corresponde a un diseño de corte transversal en 182 profesionales y 578 técnicos/administrativos. Se realizó un análisis descriptivo e inferencial, con nivel de significación 5\%. Se cuenta con la aprobación del Comité de Ética y Consentimiento Informado. Se obtuvo como resultado que el $44,9 \%$ consume medicamentos no prescritos; al comparar con situación profesional se tiene $\mathrm{OR}=1,66$, IC (1,18-2,32); en consumo de tabaco $\mathrm{OR}=1,38$, IC $(1,02-1,87)$; en consumo de alcohol $\mathrm{OR}=2,10$, IC $(1,55-2,86)$; en hipertensión arterial $\mathrm{OR}=2,25 \mathrm{IC}(1,29-3,91)$; y cefaleas $\mathrm{OR}=1,73$, IC $(1,27-2,37)$. Un alto porcentaje de automedicación, datos similares a un estudio hecho en farmacéuticos y estudiantes de Ciencias de la Salud (38,5\%). Asimismo, hay un alto riesgo en variables asociadas, concordante con estudios internacionales que señalan factores exógenos y factores endógenos asociados. Se concluye que existe alta prevalencia de automedicación y que los factores asociados (consumo de tabaco y alcohol) inciden en esta práctica. Se espera que se fomente la indagación en la salud del personal sanitario, garantizando la seguridad de las actividades y la impecabilidad en el ejercicio técnico profesional. 
Palabras Clave: Medicamentos sin prescripción, Técnicos en Salud, Automedicación, Grupo de riesgo.

\begin{abstract}
The objective of the study was to assess the situation of consumption of nonprescribed drugs by health professionals, technical/administrative staff and its associated factors. It corresponds to a cross-sectional design in 182 professionals and 578 technicians/ administratives. A descriptive and inferential analysis was performed, with a significance level of 5\%. The Committee on Ethics and Informed Consent has approved this study. It was obtained that $44.9 \%$ consumed non-prescribed medication, when comparing with professional situation, $\mathrm{OR}=1.66, \mathrm{CI}(1.18-2.32)$; in tobacco consumption $\mathrm{OR}=1.38, \mathrm{CI}$ 1.02-1.87); in alcohol consumption $\mathrm{OR}=2.10$, CI (1.55-2.86); in arterial hypertension $\mathrm{OR}$ $=2.25 \mathrm{CI}$ (1.29-3.91), and in headaches OR $=1.73$, CI (1.27-2.37). A high percentage of self-medication is shown, data similar to a study performed in pharmacists and students of Health Sciences (38.5\%). There is also a high risk in associated variables, consistent with international studies that point out exogenous factors and associated endogenous factors.

It is concluded that there is a high prevalence of self-medication and that the associated factors (smoking and alcohol consumption) influence this practice. It is hoped that the investigation into the health status of health professionals will be promoted, thus guaranteeing the safety of their activities and the impeccability in the practice of the profession.
\end{abstract}

Keywords: Nonprescription Medications, Health Technicians, Self-Medication, Risk Group.

Resumo: O objetivo do estudo foi demonstrar a utilização de medicamentos não prescritos no, saúde / profissional técnica administrativa e fatores associados. Corresponde a um desenho transversal de 182 profissionais e 578 técnicos/administrativos. análise descritiva e inferencial foi realizada com o nível de significância de 5\%. É aprovado pela Comissão de Ética e consentimento informado. $44,9 \%$ foi obtido que apresenta o consumo de medicamentos, comparando situação profissional tem $\mathrm{OR}=1,66$, CI $(1,18-2,32)$, 0 consumo de tabaco $\mathrm{OR}=1,38$, CI $(1,02-1,87)$, em álcool $\mathrm{OR}=2,10$, CI $(1,55-2,86)$, na hipertensão $\mathrm{OR}=2,25$ IC $(1,29-3,91)$, e OR = 1,73 dores de cabeça, CI $(1,27-2,37)$. Elevada percentagem de auto-medicação, semelhante a um farmacêutico de estudo e estudantes de Ciências da Saúde (38,5\%) de dados. Além disso, elevado risco associado, variáveis consistentes com estudos internacionais indicando factores exógenos associados e factores endógenos. Concluiu-se que uma elevada prevalência de auto-medicação e factores associados (consumo de tabaco e álcool), afectar esta prática. O inquérito está prevista para ser promovido a saúde dos trabalhadores de saúde, garantindo a segurança das atividades e impecabilidade em exercício técnico profissional.

Palavras-chave: Medicamentos de Balcão, Técnica de Saúde, Automedicação, Grupo de Risco. 


\section{INTRODUCCIÓN}

El problema de estudio se enmarca en el consumo de medicamentos por parte de profesionales, técnicos/administrativos de la salud y sus factores asociados, quienes, se asume, presentan mayor conocimiento y un acceso más expedito a los medicamentos, lo cual hace inferir que frente a una afección o enfermedad no realicen una consulta oportuna con el profesional referente, sino que, por el contrario, desemboquen en una conducta de consumo de medicamentos sin prescripción médica.

Esta actitud se puede explicar por la repetición de patrones de indicaciones de medicamentos realizadas previamente por el profesional y que, una vez finalizado el tratamiento, les queden medicamentos a su disposición. Frente a esto, al reiterarse esas dolencias o afecciones recurren a éstos sin la consulta profesional pertinente. De igual forma se observa el acceso que los profesionales tienen a muestras médicas, a laboratorios y a visitadores médicos. Asimismo, en el trabajo cotidiano, se establecen relaciones de confianza y contacto directo con el profesional médico, lo que facilita, por un lado, un mayor conocimiento de patologías, tratamientos y medicamentos, y por otro, la obtención de muestras o medicamentos directamente de este profesional.

En este escenario, si bien los profesionales, técnicos y administrativos de salud pueden presentar una base teórica sobre medicamentos, esta situación no está exenta de riesgos, lo cual sumado a una confianza excesiva en el conocimiento que se tiene, puede ocasionar daños colaterales para la salud.

En este sentido, la Organización Mundial de la Salud (OMS) propone directrices que apunten a una mejor estrategia sistemática de comunicación y sensibilización de todo el personal sanitario (1). Al respecto, desde el punto de vista legislativo, la venta no regulada de algunos medicamentos, como los analgésicos, es una constante que facilita su consumo sin la prescripción debida. La política farmacéutica debe asegurar la calidad de los medicamentos, fomentando su uso racional, formando parte de la agenda de salud nacional $(2,3)$. Por ello, es importante que se aúnen acciones con objetivos amplios y concretos para la población en general, proceso que puede ser facilitado a través de la organización de una política ministerial $(4,5)$.

Debido al consumo de medicamentos sin indicación médica, la modificación arbitraria de dosis, la frecuencia y tiempo de administración, lo antedicho constituye un problema de salud pública, especialmente en países desarrollados y, específicamente, en el uso de fármacos como benzodiacepinas (6). A raíz de esto, algunas naciones han instaurado que sean los farmacéuticos quienes resguarden el uso adecuado e identifiquen a personas con riesgo de abuso de medicamentos (7).

Chile no está exento de la situación planteada, por lo que en esta investigación se estudia la situación del consumo de medicamentos sin prescripción médica en profesionales y técnicos/administrativos de la salud, contexto que se aborda desde la perspectiva 
epidemiológica y social, fundamentada por su magnitud y riesgos tanto para el ente de salud como para la atención de los usuarios.

El consumo de medicamentos no prescritos por el profesional médico es un tema de creciente relevancia, y que, como se ha mencionado anteriormente, amerita la revisión de medidas y resguardos para su control y supervisión (8). Si bien esta práctica pareciera inevitable, las autoridades y profesionales de la salud deben educar a la población acerca de los riesgos y buscar estrategias que incrementen el uso seguro y eficiente de medicamentos, a través de intervenciones dirigidas al equipo de salud, farmacias y a consumidores, concientizando en evitar hacer uso de esta práctica personal (4).

Una estrategia eficiente ha sido la implementación de Guías Clínicas para promover el consumo y uso adecuado de medicamentos, como elemento central en la formación de profesionales (9-10). Sin embargo, no está exenta de inconvenientes, como el monitoreo de prácticas de prescripción en las instituciones de salud. En este aspecto, para lograr el consumo seguro y adecuado de medicamentos se debe reflexionar acerca de la normativa de exigir receta médica en toda venta de éstos, debido a que a la fecha sólo lo requieren por ley los estupefacientes y antibióticos, los cuales se encuentran regulados y controlados. No obstante, existe un número de medicamentos de venta libre, lo que ha dado lugar a una práctica cotidiana en el consumo de medicamentos no prescritos, estimada entre $43 \%$ y $55 \%$, según un estudio de Wirtz y colaboradores (11).

Conjuntamente, la decisión de ingerir medicamentos debe estar acompañada con información al respecto, tal como ocurre en países desarrollados y en algunas naciones de Latinoamérica, donde se realizan consejerías (12). Sin embargo, existe limitada inversión para incrementar el conocimiento de los consumidores y el acceso a información sobre salud y medicamentos (13).

Una investigación de magnitud y factores de consumo de medicamentos no prescritos, realizado en médicos, farmacéuticos y estudiantes de salud, efectuada por Auta y colaboradores el año 2012, señala que un 38,5\% realizaba esta práctica y que los medicamentos fueron obtenidos en farmacias o en tiendas. Entre los más utilizados se mencionan el paracetamol y antiinflamatorios no esteroideos (AINE). La experiencia previa y la no gravedad de la enfermedad fueron los dos principales factores reportados para su ingesta. En el mismo escenario, en estudiantes de farmacia, muestra que $66,0 \%$ contaba con medicamentos en su habitación, 37,1\% poseían medicamentos sobrantes de indicaciones previas como analgésicos y sólo obtenían los medicamentos con receta médica un $25,8 \%$. La práctica de consumo de medicamentos no prescritos fue del 53,2\%, sin relación significativa con el conocimiento de medicina (14).

En concordancia con lo anterior, en estudiantes universitarias de enfermería la prevalencia de esa práctica fue del 38,8\%, siendo el principal motivo el dolor autoreferido con intensidad moderada, predominantemente crónico y localizado en la cabeza, según lo estudiado por Ferreira y su equipo en el año 2011(15). 
La venta de analgésicos está muy extendida en todo el mundo, debido a que el dolor es uno de los principales motivos de consumo y, a la vez, forma parte de los medicamentos que no presentan restricción en su venta. La disposición de éstos en venta libre es una dificultad en el ámbito sanitario, facilitando el tratamiento express para trastornos o síntomas autodiagnosticados, lo que conlleva a un uso intermitente o continuo de un medicamento no prescrito $(1,2)$. Esto aumenta los riesgos de complicaciones asociadas, debido a que sus efectos secundarios tienen amplia variabilidad y su consumo excesivo podría ser perjudicial para la salud (1).

Los profesionales de la salud desempeñan un rol importante en la promoción del consumo adecuado de medicamentos prescritos y deben procurar una participación activa en la prevención del abuso de medicamentos de venta libre, así como evitar el consumo propio de medicamentos no prescritos (16).

Frente a lo anterior se plantea la inquietud de cuál es la situación de automedicación en el área sanitaria, con el objetivo de establecer la situación de consumo de medicamentos no prescritos en profesionales, técnicos/administrativos de la salud y factores asociados a esta práctica (variables sociobiodemográficas, clínicas, laborales), con el propósito de contribuir a la evidencia de una situación de salud pública local, que genere instancias de mejoras de políticas en el área.

\section{MATERIAL Y MÉTODO}

Estudio bajo el paradigma positivista, diseño de corte transversal, en un hospital de alta complejidad, año 2016. Muestra no probabilística por conveniencia, de una población de 1254 (509 profesionales no médicos y 745 técnicos/administrativos). Cumplieron con los criterios de selección, aceptaron participar en el estudio y firmaron Formulario de Consentimiento Informado 760 de ellos (182 profesionales no médicos y 578 técnicos/administrativos). Recolección de la información realizada a través del cuestionario "Automedicación en Profesionales y Técnicos de la salud" de Rocha A, Harter R, Rotenberg, adaptado para el estudio (17). Aplicación del instrumento en el establecimiento de salud estudiado. Se realizó análisis descriptivo y de asociación entre las variables sociodemográficas, laborales y clínicas con consumo de medicamentos no prescritos. Nivel de significación 5\%; se usó programa STATA 11.0. Se contó aprobación del Comité de Ética del Servicio de Salud Araucanía Sur.

\section{RESULTADOS}

De las 760 personas que aceptaron participar del estudio, $24,0 \%$ eran profesionales no médicos; la edad promedio fue de 36,5 $\pm 12,0$ años, con un rango de 18 a 70 años. El 23,2\% eran hombres, mapuches $19,6 \%$, sin pareja $60,3 \%$, urbanos $6,7 \%$, con problemas económicos 34,2\%, trabajando en sistema de turnos 57,4\%, realizando turnos extras $60,8 \%$, con hábitos tabáquicos $32,9 \%$, de alcohol 35,9\% y los que estaban conforme con su trabajo 93,3\%. De las variables clínicas, el 9\% presentó hipertensión arterial, con diabetes mellitus $5,8 \%$, con hospitalizaciones previas $46,2 \%$, sin presencia de afecciones $37,2 \%$ y $\sin$ 
presencia de alteraciones psiquiátricas 88,9\%. El 67,2\% declaró tener ingresos menores de $\$ 500$ mil pesos, el $19,8 \%$ entre $\$ 500$ y $\$ 900$ mil pesos y $13 \%$ más de $\$ 900$ mil pesos. El consumo de medicamentos no prescritos fue de $44,9 \%$.

Al comparar consumo de medicamentos no prescritos con las variables sociodemográficas se encontró diferencias significativas con: situación profesional, donde los profesionales no médicos tienen 1,66 veces más probabilidad de consumirlos; en consumo de tabaco, donde los que fuman tienen 1,38 veces más probabilidad consumirlos; en consumo de alcohol, donde los que beben tienen 2,10 veces más probabilidad consumirlos. No hay diferencias significativas con sexo, etnia, situación conyugal, residencia, ingreso mensual y con problemas económicos (Tabla I).

Tabla I. Consumo de medicamentos en profesionales, técnicos/administrativos de la salud, según variables sociodemográficas. Hospital alta complejidad año 2016.

\begin{tabular}{l|l|c|c|c|c}
\hline Variables & & $\mathbf{n}$ & $\mathbf{\%}$ & OR & IC \\
\hline Sexo & Mujer (582) & 261 & 44,8 & & \\
& Hombre (176) & 79 & 44,9 & 1,00 & $0,71-1,41$ \\
\hline Etnia & No mapuche (609) & 268 & 44,0 & & \\
& Mapuche(149) & 72 & 48,3 & 1,19 & $0,83-1,70$ \\
\hline Situación & Con pareja (301) & 125 & 41,5 & & \\
Conyugal & Sin pareja (457) & 215 & 47,1 & 1,25 & $0,93-1,68$ \\
\hline Situación & Tec/Adm. (576) & 241 & 41,8 & & \\
Profesional & Prof no médico (182) & 99 & 54,4 & 1,66 & $1,18-2,32$ \\
\hline Consumo & Sin consumo (509) & 215 & 42,2 & & \\
de tabaco & Consumo (249) & 125 & 50,2 & 1,38 & $1,02-1,87$ \\
\hline Consumo & Sin consumo (486) & 186 & 38,3 & & \\
de alcohol & Consumo (272) & 154 & 56,6 & 2,10 & $1,55-2,86$ \\
\hline Residencia & Urbano (707) & 317 & 44,8 & & \\
& Rural (51) & 23 & 45,1 & 1,01 & $0,57-1,79$ \\
\hline Ingreso & Menor a 500 (510) & 218 & 42,8 & & \\
Mensual (miles) & Entre 500-900 (149) & 70 & 47,0 & 1,19 & $0,82-1,71$ \\
& Mayor a 900 (99) & 52 & 52,5 & 1,48 & $0,96-2,28$ \\
\hline Problemas & Sin problemas (498) & 220 & 44,2 & & \\
económicos & Con problemas (260) & 120 & 46,2 & 1,08 & $0,80-1,46$ \\
\hline
\end{tabular}

Fuente: Elaboración propia (2016)

Con las variables laborales: sistema de turno, realiza turnos extras, conformidad con el trabajo y su calificación, no se asocia significativamente (Tabla II). 
Tabla II. Consumo de medicamentos en profesionales, técnicos/administrativos de la salud, según variables laborales. Hospital alta complejidad, Chile 2016.

\begin{tabular}{l|l|c|c|c|c}
\hline \multicolumn{1}{l|}{ VARIABLES } & $\mathbf{n}$ & $\mathbf{\%}$ & OR & IC \\
\hline $\begin{array}{l}\text { Sistema } \\
\text { de turno }\end{array}$ & No turnos (324) & 138 & 42,6 & & \\
\hline Realiza & Con turnos (434) & 202 & 46,5 & 1,17 & $0,88-1,57$ \\
turnos extras & No realiza (297) & 122 & 41,1 & & \\
\hline $\begin{array}{l}\text { Conformidad } \\
\text { con el trabajo }\end{array}$ & Realiza (461) & 218 & 47,3 & 1,29 & $0,96-1,73$ \\
\hline $\begin{array}{l}\text { Conforme con } \\
\text { calificación }\end{array}$ & Conforme (707) & 26 & 51,0 & & \\
\hline
\end{tabular}

Fuente: Elaboración propia (2016)

Con las variables clínicas se encontraron diferencias significativas con: hipertensión arterial, donde los que no presentan hipertensión tienen 2,25 veces más probabilidad de consumirlos; afección clínica, donde los que presentan alguna afección tienen 1,74 veces más probabilidad de consumirlos; cefaleas, donde los que las presentan tienen 1,73 veces más probabilidad de consumirlos. No hay diferencias significativas con diabetes mellitus, hospitalización previa, alteraciones psíquicas, gastritis, insomnio, otras alteraciones (Tabla III).

Tabla III. Consumo de medicamentos en profesionales, técnicos/administrativos de la salud, según variables clínicas. Hospital Alta Complejidad. Chile, 2016.

\begin{tabular}{l|l|c|c|c|c}
\hline \multicolumn{1}{l|}{ VARIABLES } & $\mathbf{n}$ & $\mathbf{\%}$ & OR & IC \\
\hline Hipertensión & Presenta (68) & 19 & 27,9 & & \\
Arterial & No presenta (689) & 321 & 46,6 & 2,25 & $1,29-3,91$ \\
\hline Diabetes & No presenta (714) & 322 & 45,1 & & \\
Mellitus & Presenta (44) & 18 & 40,9 & 0.84 & $0,45-1,57$ \\
\hline Hospitalización & No presenta (408) & 189 & 46,3 & & \\
previa & Presenta (350) & 151 & 43,1 & 0.87 & $0,66-1,17$ \\
\hline Alteraciones & No presenta (674) & 295 & 43,8 & & \\
psiquiátricas & Presenta (84) & 45 & 53,6 & 1,48 & $0,94-2,34$ \\
\hline Gastritis & No Presenta (594) & 256 & 43,1 & & \\
& Presenta (164) & 84 & 51,2 & 1,39 & $0,98-1,96$ \\
\hline Insomnio & No Presenta (636) & 280 & 44,0 & & \\
& presenta (122) & 60 & 49,2 & 1,23 & $0,83-1,81$ \\
\hline Cefaleas & No presenta (520) & 211 & 40,6 & & \\
& Presenta (238) & 129 & 54,2 & 1,73 & $1,27-2,37$ \\
\hline Otras & No presenta (616) & 277 & 45,0 & & \\
alteraciones* & Presenta (142) & 63 & 44,4 & 0.98 & $0,68-1,41$ \\
\hline
\end{tabular}

*Hipertiroidismo, mialgias, dermatitis, alergias.

Fuente: Elaboración propia (2016) 


\section{DISCUSIÓN}

En nuestro estudio 23,2\% de los hombres consume fármacos no prescritos; investigaciones en farmacéuticos y estudiantes de ciencias de la salud reportan 38,5\% de automedicación y los medicamentos más utilizados son el paracetamol y antiinflamatorios (AINE), siendo la experiencia previa y la no gravedad de la enfermedad los factores reportados para automedicación (18). Otro estudio evidenció automedicación en 53,2\%, sin relación significativa entre poseer conocimientos de medicina y automedicación (14). En nuestro estudio el consumo de medicamentos no prescritos se asoció a situación profesional, consumo de tabaco, alcohol, hipertensión y cefaleas. Al respecto, variables de orden cognitivo-sociales explican por qué una persona adopta o no un comportamiento particular en favor o en contra de su propia salud, lo que podría explicar, por ejemplo, que se observen médicos neumólogos fumadores (19)

En este escenario, un estudio señala como factores asociados a la automedicación en el personal técnico de enfermería, un $88,89 \%$ de origen exógeno y un $47,22 \%$ factores endógenos. Como factor exógeno se señala la venta libre con $80,56 \%$ y medios de publicidad 38,89\%. Dentro del factor endógeno, el $88,89 \%$ se menciona el factor tiempo (20).

En nuestro estudio se observan como factores de riesgo la conformidad con el trabajo, el sistema de turnos o la realización de turnos extras, la situación conyugal, la residencia, el ingreso mensual y los problemas económicos, resultados similares a los obtenidos por Llanos y colaboradores (21).

Los resultados expuestos muestran una realidad local de la situación de consumo de medicamentos sin prescripción médica, por lo que se insta a buscar estrategias que colaboren en su disminución para permitir un equilibrio de la salud física y mental. Los profesionales que no asuman un estilo de vida saludable representan un problema adicional para la salud de los usuarios y organizaciones sanitarias.

Se espera que a través de esta investigación se fomente la indagación en aspectos propios de la salud del personal sanitario, garantizando la seguridad de las actividades y el logro de impecabilidad en el ejercicio técnico profesional. Sin embargo, aún persisten retos importantes sobre el consumo de medicamentos, incluyendo la utilización de sistemas de monitoreo y evaluación, limitante en este estudio.

\section{CONCLUSIONES}

Se concluye que existe alta prevalencia de automedicación y que los factores asociados (consumo de tabaco y alcohol), inciden en esta práctica. Se espera que se fomente la indagación en la salud del personal sanitario, garantizando la seguridad de las actividades y la impecabilidad en el ejercicio técnico profesional.

Se sugiere continuar desarrollando investigaciones, respecto del consumo de medicamentos, dada la valorización del contenido, la importancia y relevancia de éste, que 
impacta al paciente en su pronóstico a largo plazo. Situación que hace indudable la detección temprana y la coordinación de los miembros del equipo de salud

Se deben desarrollar estrategias de manejo eficaces con la finalidad, de garantizar una atención de calidad. Se necesitan investigaciones que puedan medir los efectos relacionados con el consumo de medicamentos y su impacto a largo plazo.

\section{AGRADECIMIENTOS}

A la Dirección de Investigación de La Universidad de La Frontera, Chile, por el financiamiento al Proyecto DIUFRO 12-0039.

\section{REFERENCIAS BIBLIOGRÁFICAS}

1. World Health Organization. Everybody's business: Strengthening health systems to improve health outcomes. WHO's framework for action. Geneva, WHO.

2. Organización Mundial de la Salud. Cómo desarrollar y aplicar una política farmacéutica nacional. 2da edición. Ginebra, Suiza: OMS, 2002.

3. Fundación Mexicana para la Salud, A.C. trabajando por la salud de la población: Propuestas de política para el sector farmacéutico. Versión para el diálogo. México, DF: Funsalud, 2011. Disponible en: http://funsalud.org.mx/portal/wpcontent/uploads/2013/08/Doc-PolPublSFarm-vFDigital-060511.pdf

4. Wirtz V, Dreser A \& Heredia-Pi Il. Retos y oportunidades para el desarrollo de la política farmacéutica nacional en México Salud Púb Mex 2013; 55(3):329-336. [consultado 2016 noviembre 4]. Disponible en: http://www.saludyfarmacos.org/boletinfarmacos/

5. Organización Panamericana de Salud Pública. Políticas Farmacéuticas Nacionales y Subregionales. 2011 [sitio en Internet]. Washington: OPS [consultado 2016 noviembre 4].

Disponible

en:

http://new.paho.org/hq/index.php?option=com_content\&task=view\&id=1903\&Itemid= 1177

6. López A, Aroche A, Bestard J \& Ocaña N. Uso y abuso de las benzodiacepinas. 2010. Disponible en: http://bvs.sld.cu/revistas/san/vol_14_4_10/san17410.htm

7. BOE. BOLETÍN OFICIAL DEL ESTADO. 2012. [consultado 2016 noviembre 4]. Disponible en: https:/www.boe.es/boe/dias/2012/12/29/pdfs/BOE-A-2012-15711.pdf

8. Ministerio de Economía de Chile, Proyecto de Ley Venta de medicamentos fuera de Farmacias, publicado el 21 de marzo de 2012. [consultado 2016 noviembre 4]. Disponible en: http://www.economia.gob.cl/proyecto-de-ley-mejor-acceso-amedicamentos/

9. Organización Mundial de la Salud. Cómo desarrollar y aplicar una política farmacéutica nacional. 2da edición. Ginebra, Suiza: OMS, 2002.

10. Laing R, Hogerzeil H \& Ross-Degnan D. Ten recommendations to improve use of medicines in developing countries. Health Policy Plan 2001; 16(1):13-20. [consultado 2016 noviembre 4]. Disponible en: http://www.ncbi.nlm.nih.gov/pubmed/11238425

11. Wirtz V, Reich M, Leyva R \& Dreser A. Medicines in México, 1990-2004: a systematic review of research on access and use. Salud Pub Mex 2008; 50:S470-S479. 
12. ANMAT. Prospectos: advertencia por incumplimiento de normativas vigentes [documento en Internet]. Buenos Aires, ANMAT, 2008.[consultado 2016 noviembre 4] Disponible http://www.anmat.gov.ar/webanmat/Comunicados/Comunicado_Prospecto_Incumplimi entoNormativas.pdf

13. González P \& Barraza-Lloréns M. Trabajando por la salud de la población: Propuestas de política para el sector farmacéutico. Versión para el diálogo. México, DF: Funsalud, 2011.

14. Auta A; Banwat S, Sariem C, Shalkur D, Nasara B, Atuluku MJ Young Pharm. Medicines in Pharmacy Students' Residence and Self-medication Practices. 2012; 4(2):119-23. [consultado 2016 noviembre 4] Disponible en: http://www.ncbi.nlm.nih.gov/pmc/articles/PMC3385216/

15. Ferreira L; Damázio da Silva C; Carvalho G; Faleiros F; Varanda L. Prevalencia y caracterización de la práctica de automedicación para alivio del dolor entre estudiantes universitarios de enfermería Rev. Latino-Am. Enfermagem. 2011; 19 (2) [consultado 2016 noviembre 4] Disponible en: https://www.revista-portalesmedicos.com/revistamedica/automedicacion-riesgo-para-la-salud/3/

16. Yakugaku T. The Pharmacist As Gatekeeper Of Prescription Drug Abuse: Return To "Community Scientists" 2016; 136 (1): 79-87. [consultado 2016 noviembre 4] Disponible en: https://www.jstage.jst.go.jp/article/yakushi/136/1/136_15-002283/_article

17. Rocha R, Harter A \& Rotenberg R. Automedicação entre os trabalhadores de enfermagem de hospitais públicos. Rev. Latinoam. Enfermagem. 2009; 17(6). Universidade de São Paulo. São Paulo, Brasil. [consultado 2016 septiembre 30]. Disponible en: http://redalyc.uaemex.mx/src/inicio/ArtPdfRed.jsp?iCve=281421912014

18. Abay S \& Amelo W. Assessment of self-medication practices among medical, pharmacy, and health science students in gondar university, ethiopia. J Young Pharm. 2010; 2(3):306-10. [consultado 2016 noviembre 4] Disponible en: http://www.ncbi.nlm.nih.gov/pubmed/17565044

19. Conner M. The role of social cognition in health behaviours en: Conner M, Norman P, editors. Predicting Health Behaviour: Research Practice with Social Cognition Models. 1993. [consultado 2016 septiembre 30]. Disponible en: http://onlinelibrary.wiley.com/doi/10.1111/j.15591816.1998.tb01685.x/abstract;jsession id $=8741$ CC654617A0425E1C207AD8836C79.f02t04?deniedAccessCustomisedMessa $\mathrm{ge}=$ \&userIsAuthenticated $=$ false

20. Higa S. Los factores asociados a automedicación en personal técnico de enfermería en los servicios de medicina.2015 [consultado 2016 septiembre 30]. Disponible en: http://cybertesis.unmsm.edu.pe/handle/cybertesis/4764

21. Llanos Zavalaga, L et al. Automedicación en cinco provincias de Cajamarca. Rev Med Hered 2001; 12: 127-133. [consultado 2016 noviembre 20]. Disponible en: http://www.scielo.org.pe/scielo.php?script=sci_arttext\&pid=S1018130X200100040000 $4 \& \operatorname{lng}=\mathrm{es} \& \mathrm{nrm}=$ iso 